\title{
The effect of subionospheric propagation on whistlers recorded by the DEMETER satellite - observation and modelling
}

\author{
O. E. Ferencz ${ }^{1}$, Cs. Ferencz ${ }^{1}$, P. Steinbach ${ }^{2}$, J. Lichtenberger ${ }^{1}$, D. Hamar ${ }^{1}$, M. Parrot ${ }^{3}$, F. Lefeuvre ${ }^{3}$, and \\ J.-J. Berthelier ${ }^{4}$ \\ ${ }^{1}$ Space Research Group, Eötvös University, (Inst. of Geogr. and Earth Sci.), 1117 Budapest, Pázmány P.s. 1/A, Hungary \\ ${ }^{2}$ MTA-ELTE Research Group for Geology, Geophysics and Space Sciences, 1117 Budapest, Pázmány P.s. 1/A, Hungary \\ ${ }^{3}$ LPCE/CNRS 3A Avenue de la Recherche, 45071, Orléans, Cedex 2, France \\ ${ }^{4}$ CETP/IPSL, Observatoire de Saint Maur, 4, Avenue de Neptune, Saint Maur des Fossés, 94107, France
}

Received: 14 December 2006 - Revised: 16 March 2007 - Accepted: 4 April 2007 - Published: 4 June 2007

\begin{abstract}
During a routine analysis of whistlers on the wideband VLF recording of the DEMETER satellite, a specific signal structure of numerous fractional-hop whistlers, termed the "Spiky Whistler" (SpW) was identified. These signals appear to be composed of a conventional whistler combined by the compound mode-patterns of guided wave propagation, suggesting a whistler excited by a lightning "tweek" spheric. Rigorous, full-wave modelling of tweeks, formed by the long subionospheric guided spheric propagation and of the impulse propagation across an arbitrarily inhomogeneous ionosphere, gave an accurate description of the $\mathrm{SpW}$ signals. The electromagnetic impulses excited by vertical, preferably CG lightning discharge, exhibited the effects of guided behaviour and of the dispersive ionospheric plasma along their paths. This modelling and interpretation provides a consistent way to determine the generation and propagation characteristics of the recorded SpW signals, as well as to describe the traversed medium.
\end{abstract}

Keywords. Electromagnetics (Guided waves) - Radio science (Ionospheric propagation) - Space plasma physics (Experimental and mathematical techniques)

\section{Introduction}

Lightning discharges in the neutral atmosphere radiate ultra wide-band (UWB) electromagnetic signals. These short impulses may directly enter the plasma environment of the Earth, followed by propagation in this dispersive, anisotropic medium and appear as conventional whistlers in the ULFVLF frequency range (Helliwell, 1965) or as TiPPs in the HF band (Holden et al., 1995; Ferencz et al., 2001) on electric and/or magnetic recordings. The low frequency part of the radiated power, depending on the direction of the lightning

Correspondence to: O. E. Ferencz

(spacerg@sas.elte.hu) current, may propagate large distances with considerable low attenuation, on the order of a few $\mathrm{dB}$ per thousand $\mathrm{km}$ of the path (Taylor, 1960; Burke and Jones, 1992) in the waveguide formed by the highly conducting solid Earth and by the almost perfect conductor lower ionosphere. The guided propagation dictates that distinct propagation modes, allowed and ruled by this Earth-ionosphere waveguide (EIWG), appear out of necessity on spheric impulses. Distant propagated spheric signals have often been observed and are well known as tweeks (Helliwell, 1965). Spherics and tweeks are perfect tools to analyze the exciting lightning discharge, its location and the radiated wideband waveform. They provide a basis to monitor the conditions of the subionospheric VLF propagation, for instance, the virtual ionospheric "height" or the incident angle of the signal at the ground receiver. This aspect has been utilized by numerous investigations, applying single frequency (monochromatic), multiple mode descriptions of the wave propagation (Budden, 1961; Shvets and Hayakawa, 1998), yielding information about the spatial and temporal character of the ionization in the mesosphere (Yedemsky et al., 1992; Hayakawa et al., 1994; Cummer et al., 1998).

A part of the energy propagating in the EIWG may enter the plasma environment of the Earth, start to propagate in an anisotropic, inhomogeneous, dispersive medium, formed by the ionosphere. These short path signals can be recorded on board satellites flying, for example, at LEO altitudes. In our study during analysis of wideband VLF waveforms recorded by the French DEMETER LEO satellite we have observed upward propagating whistlers exhibiting a strange shape. Based on our previous studies in the field of theoretical wave propagation modelling (Ferencz et al., 2001; Ferencz, 2004a), the propagation of an arbitrary shaped UWB signal in the EIWG and the subsequent path across the inhomogeneous, anisotropic ionosphere up to the satellite altitude was possible to describe analytically.

We give here the first report about observations of specific fractional-hop whistlers, recorded on board the LEO

Published by Copernicus GmbH on behalf of the European Geosciences Union. 


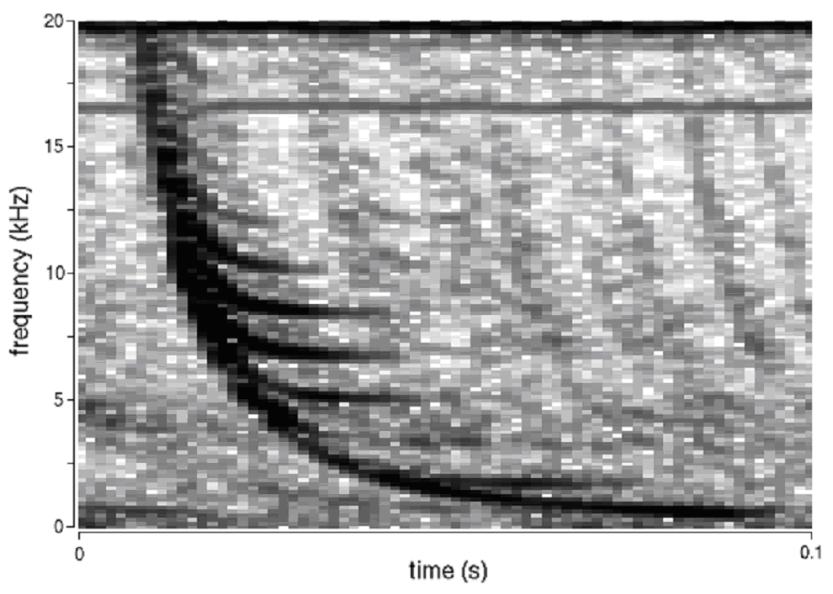

Fig. 1. Spiky whistler in DEMETER ICE burst mode VLF recording (orbit: 1242 up, sensor: E34, date: 25 September 2004, 21:30:33.2 UT, satellite position: $34.7 \mathrm{~N}$; 5.0 E, altitude: 702.3, L: 1.49).

DEMETER satellite. According to our investigation, these signals were excited by tweeks in the lower ionosphere, showing the mode pattern of their origin, termed hereafter as "Spiky Whistlers" (SpW). After a short overview of the analyzed onboard data set (Sect. 2) a preliminary description of the observed phenomenon is given in Sect. 3. The qualitative picture is supported and confirmed by accurate modelling and interpretation, detailed in Sect. 4. Results of numerical simulations of recorded SpWs, applying the full-wave solution for guided subionospheric propagation, followed by the wave propagation in an arbitrarily inhomogeneous ionospheric plasma, are given in Sect. 5.

\section{Onboard data set of the investigation}

With the launch of the DEMETER microsatellite in 2004 an important and new type of systematic monitoring of the electromagnetic environment around the planet Earth has started in the ULF-VLF to HF frequency bands (http://demeter. cnrs-orleans.fr/). On board the DEMETER satellite two electromagnetic wave experiments are in operation which are most relevant to the investigation reported in this paper: the ICE electric field instrument (Berthelier et al., 2006), operating in the DC-HF bands and the IMSC magnetic field experiment (Parrot et al., 2006), operating in the VLF band. According to the primary scientific objectives of the mission, continuous "burst" mode wave experiments (acquisition of the detailed signal waveform and power spectra) are conducted on board, restricted to previously selected, seismically active regions of the Earth, while only the power spectra is transmitted otherwise in the "survey" mode to the ground. In our detailed signal analysis the burst mode waveform recordings were used. Examples, illustrating the observed phenomenon below, are conventional FFT spectra of the recorded waveforms (window size: $6.4 \mathrm{~ms}$, overlap: $78 \%$ ). For a better comparison of the spacecraft data and of ground-based data set (broadband VLF recordings in Hungary and at the ground segment of the DEMETER mission in France, available regional lightning data), the majority of the systematically investigated burst data has been selected along European passes (every pass in a 7-month period between August 2004-February 2005), although a reasonable volume of analyzed data stem from satellite paths, choosen randomly but globally.

\section{The observed signal structure}

Systematic analysis of the VLF burst recording of the DEMETER satellite yielded the observation of a remarkable, interesting signal pattern on numerous whistlers. According to their dispersion values $\left(D_{0}\right)$, these signals are upward propagating, short-path fractional-hop whistlers, which crossed a major part of the ionosphere before reaching the satellite at its LEO (approx. $710 \mathrm{~km}$ altitude). These specific whistlers are exhibiting a compound, "spiky" signal structure; see example in Fig. 1.

"Spiky Whistler" (SpW) cases have been only recognized on detailed (high temporal resolution) broadband VLF spectra. SpWs are merged with other conventional fractional-hop ones with similar dispersion, as shown in Fig. 2. The spiky feature of this phenomenon and the asymptotic behaviour of the branching signal parts are obvious consequences of guided propagation. The occurrence of the intense, welldefined $\mathrm{SpW}$ cases are rather infrequent, however, in certain periods almost all fractional-hop whistlers seem to be SpW. (This rare occurrence may be responsible for the SpW phenomenon being not recognized and described before.)

Based on the signal analysis of more than $42 \mathrm{~h}$, burst the VLF recording the average rate of strong $\mathrm{SpWs}$ relative to the conventional whistler occurrence rate is of the order of a few percent. The overall rate of SpWs seems to follow the usual whistler rate variation with season and hour of the day. According to the quasi Sun-synchronous orbit of the DEMETER, passes fall in the morning and evening hours in local time. SpW signals, like the conventional whistlers, appear more pronounced in the evening recordings. Note that a thorough investigation of a subset of the systematically analysed data set suggests that the weak appearance of this modal trace splitting is, as expected, often more frequent. Accurate statistics of $\mathrm{SpW}$ rates will be present in a later phase of our investigation, provided by the automatic whistler detection procedure (Lichtenberger et al., 2001), enhanced to classify SpW signals separately.

Asymptotes of the branches are harmonics of the lowest asymptote frequency. The latter values fall in the frequency range $1.65-2 \mathrm{kHz}$, the same as the boundary frequency of the EIWG, ruled by the "ionospheric height". Although many 


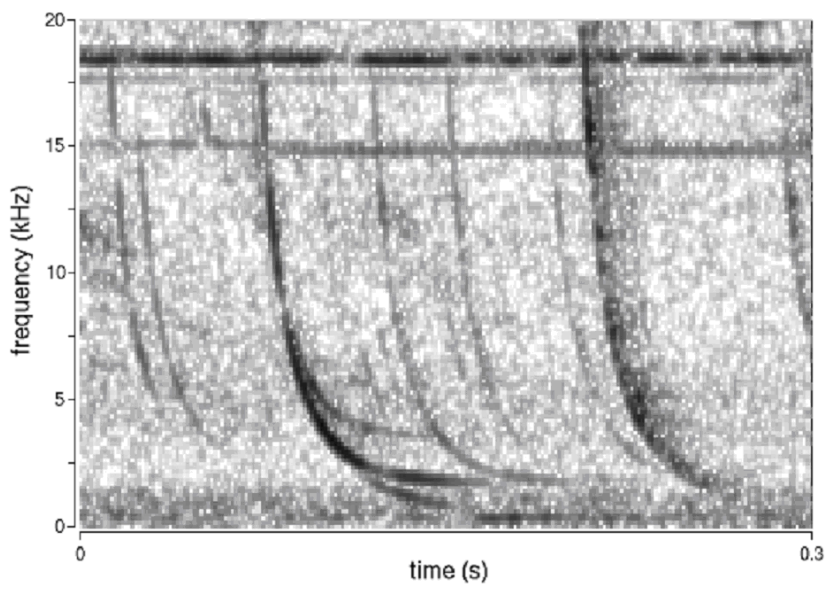

Fig. 2. Fractional hop whistler traces, exhibiting the spiky feature has a similar dispersion to surrounding, conventional ones (orbit: 683 up, sensor: E34, date: 18 August 2004, 10:37:09.8 UT, satellite position: $31.5 \mathrm{~S}$; 179.2 E, altitude: 733.3, L: 1.7).

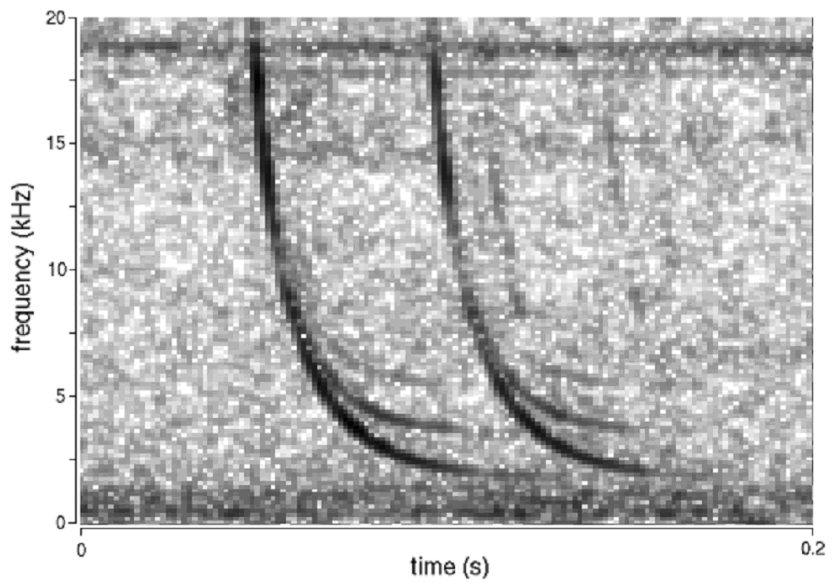

Fig. 3. Incomplete sequence of the mode pattern on "spiky whistlers", observed in DEMETER burst VLF data. The highest observable harmonic is the 3rd. Noteworthy that the zeroth-order, basic mode is also missing in this example (orbit: 683 up, sensor: E34, date: 18 August 2004, 10:36:38.8 UT, satellite position: 33.3 S ; 179.7 E, altitude: 734.2, L: 1.78).

SpW instances showed a pattern of multiple branches on FFT spectra, in contrary to our expectations based on theoretical consideration, SpWs most typically show only a fragmentary sequence of the harmonic branches (see, e.g. in Fig. 2 or Fig. 3). Moreover, this incompleteness is occasional in its character. The most often occurring variation of the SpW signals is the one with only the lowest frequency branch pattern, without the higher harmonics (Fig. 4).

Beside this, fractional-hop whistlers often show a single branching trace at higher frequencies with asymptotes in the range between $8 \mathrm{kHz}-17 \mathrm{kHz}$. They may be interpreted as anomalistic SpW cases, illustrated in Fig. 5. This

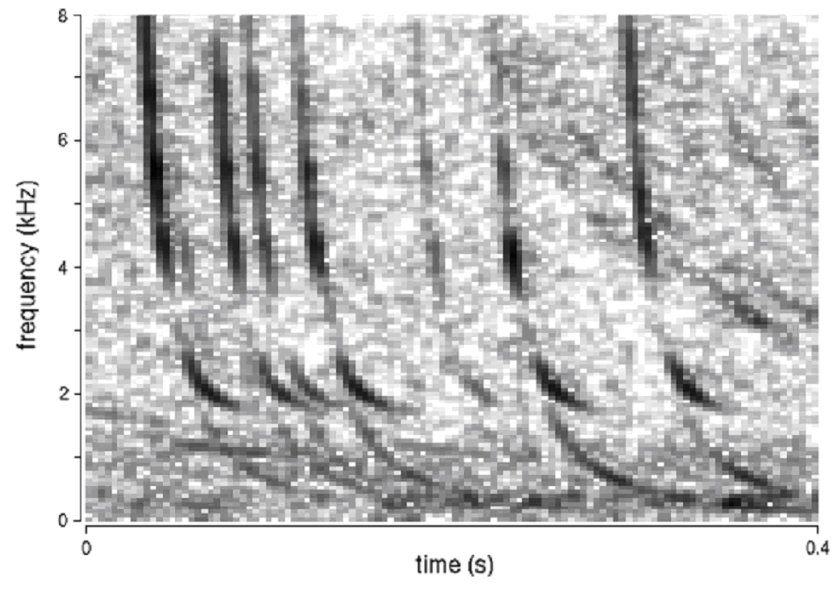

Fig. 4. Series of the lowest (transversal electromagnetic mode, TEM) and the first mode pattern on short-path fractional-hop whistlers without higher modes, seen in the ICE electric field data of the DEMETER satellite (orbit: 1430 up, sensor: E34, date: 8 October 2004, 19:48:49.3 UT, satellite position: $40.9 \mathrm{~N} ; 29 \mathrm{E}$, altitude: 713.0, L: 1.73).

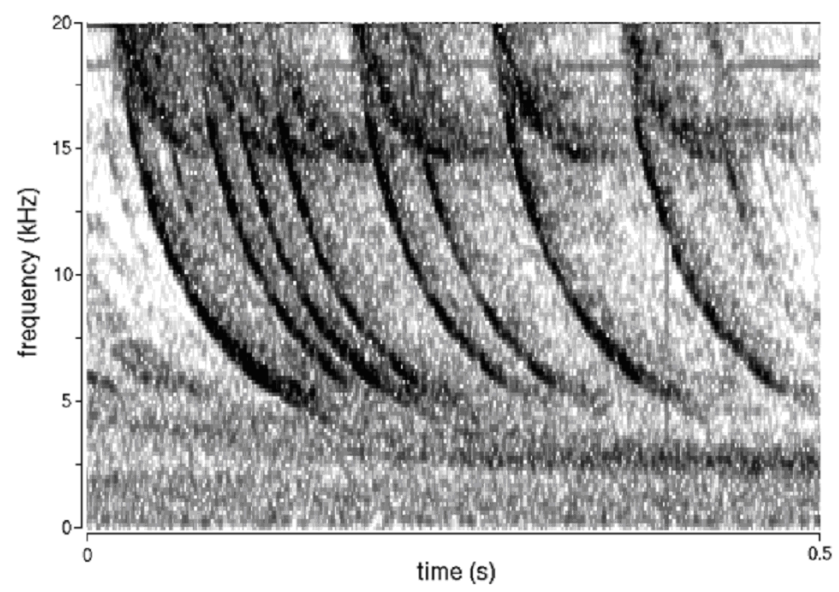

Fig. 5. Anomalistic SpW series, exhibiting single mode pattern with asymptotes at a higher part of the recorded bandwidth are often observable on the DEMETER VLF burst recordings (orbit: 2623 up, sensor: E12, date: 29 December 2004, 20:35:55.1 UT, satellite position: $36.3 \mathrm{~N}$; $18.5 \mathrm{E}$, altitude: 701.4, L: 1.51).

sort of branching can be explained as a single guided mode, leaking out of the EIWG, together with the fractional-hop whistler, or formed during the ionospheric traverse. The latter scene assumes a mode-dependent (mode-selecting) outcoupling mechanism for the VLF impulses in the lower plasmasphere or at the lower boundary of the ionosphere. Similarly to other fractional-hop whistlers, SpW signals are often seen together with ion-whistlers, propagated contemporaneously. 


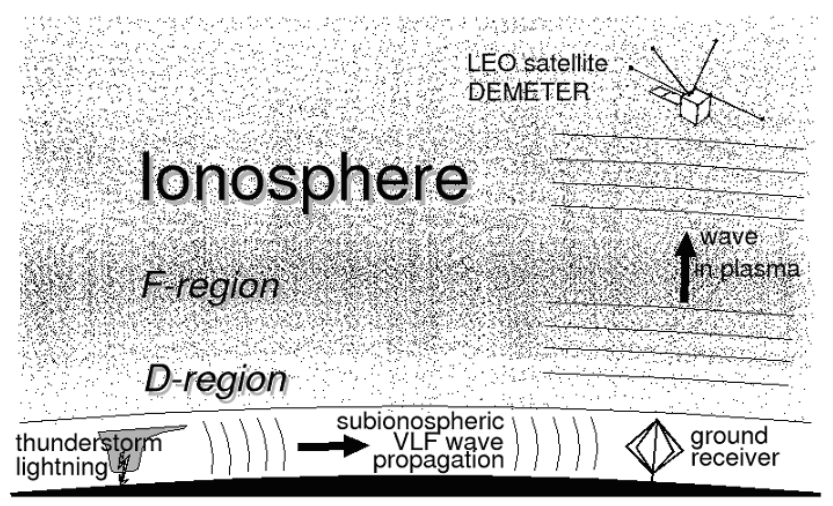

Fig. 6. Schematic of tweek generation in the EIWG and a subsequent fractional hop whistler propagation across the inhomogeneous ionosphere to a LEO satellite.

\section{Modelling and interpretation, theoretical background}

The obvious visual similarity between tweek and SpW signals and the frequencies of the lowest asymptote of the SpW branches on the FFT spectra made it reasonable to attempt modelling this phenomenon as a fractional-hop whistler, excited by a tweek at the lower ionosphere. Two consecutive segments of such a wave propagation path in different media is sketched in Fig. 6.

The general, exact full-wave solutions of Maxwell's equations for guided propagation of short, UWB impulses (Ferencz, 2004a; Ferencz and Ferencz, 2004), as well as for UWB signal propagation in linear, inhomogeneous magnetoionic media (Ferencz et al., 1996, 2001; Ferencz, 2005), were applied in this investigation.

After a short theoretical consideration on the relation between the lightning discharge direction and the type of excited signal, a description of the applied analytical solutions is given in this section.

If the source current density (the channel of the unspecified lightning discharge in the neutral atmosphere) is parallel to the lower "boundary" of the ionosphere, this kind of source generates directly a UWB signal (an upward propagating whistler) in the magnetoionic medium, reaching the LEO satellite and propagating through the plasmasphere (Ferencz et al., 2001). This is typical in the case of cloudto-cloud lightnings, and of large horizontal discharges in mesoscale convective complexes. If the source current density is closely perpendicular to the boundary surfaces of the Earth and the plasma environment, then this source will not directly excite the signal in the magnetoionic medium above at all, however, it generates guided wave modes propagating in the EIWG (Ferencz, 2004a). This happens predominantly by cloud-to-ground lightnings. (Note that the above description captures only one fundamental geometric aspect of impulse generation by lightnings in the atmosphere. Both the orientation and the linear character of a tortuous discharg-

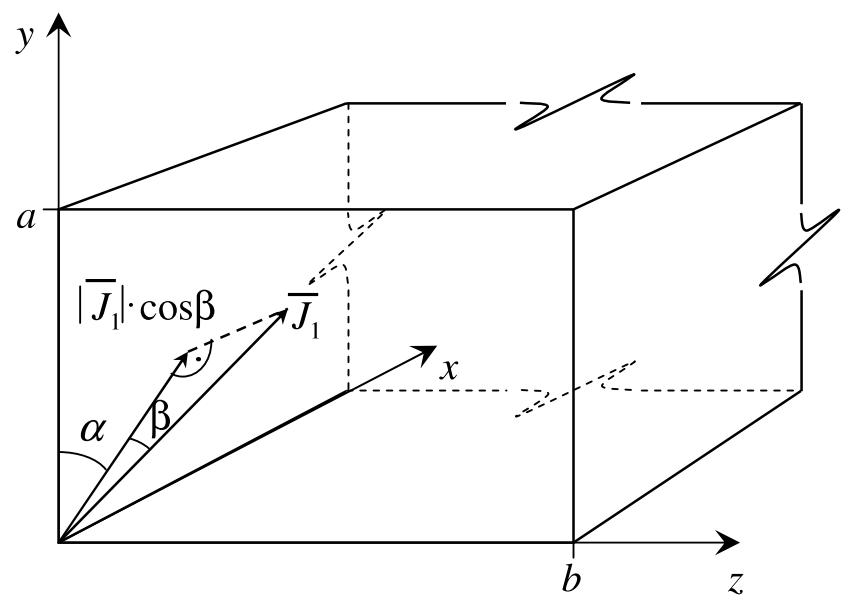

Fig. 7. The simplified geometric model applied for the UWB description of the guided wave (tweek) propagation in the EIWG.

ing channel are just usual model simplifications.) As many satellite observations and ground lightning data prove, farther from the source the propagating guided signal can enter the ionosphere and start to propagate in the magnetoionic medium. (There is no coherent description about the way, how a portion of the ELF-VLF energy, propagating along the surface can exit the EIWG. Inhomogenities in the mesosphere, at the lower "boundary" of the ionosphere may play substantial role in this process.)

In model calculations it may be advantageous to choose the Dirac-delta functional as the source signal, since this way the excited signal itself serves the "transfer function" (e.g. Simonyi, 1963; Ferencz et al., 1996, 2001), characterizing the propagation: the medium traversed by the signal and the boundary conditions. In a general full-wave solution the option to choose other sources is also possible, e.g. source with spectral character similar to natural lightnings, such as decreasing power density above $10 \mathrm{kHz}$ or finite-length impulses, etc.

If we follow the process of forming an $\mathrm{SpW}$ in the plasmasphere as a lightning excited impulse, see in Fig. 6, modelling the development of a tweek waveform is needed at first.

The general model geometry of the wave propagation, applied here to describe the waveguide formed by the Earth's surface and the rectangular lower ionosphere, infinite in $x$ or in the $x$ and $z$ directions, with perfect conducting horizontal parallel plane boundaries, are illustrated in Fig. 7. In the presented applications the waveguide is filled by a vacuum. The orientation of the arbitrarily directed lightning discharge, represented by the $\boldsymbol{J}_{1}$ source current density, is given by the angles $\alpha$ and $\beta$, the propagation is directed along the $x$ axis. The "height" of the ionosphere is set with variable $a$.

The general form of the excitation is given as:

$$
\begin{gathered}
\overline{\boldsymbol{J}}_{1}=J_{1 x} \cdot \overline{\boldsymbol{i}}+J_{1 y} \cdot \overline{\boldsymbol{j}}+J_{1 z} \cdot \overline{\boldsymbol{k}} \\
\left|\overline{\boldsymbol{J}}_{1}\right|=\delta(t) \cdot \delta(x) \cdot B_{1}(y) \cdot B_{2}(z),
\end{gathered}
$$


where $\overline{\boldsymbol{i}}, \overline{\boldsymbol{j}}$ and $\overline{\boldsymbol{k}}$ are the orthogonal components of the $\boldsymbol{e}$ unit vector, $\delta$ is the Dirac-delta functional (distribution) and $B_{1}(y)$ and $B_{2}(z)$ are envelope functions containing the source boundary conditions of the model:

$B_{1}(0)=B_{1}(a)=B_{2}(0)=B_{2}(b) \equiv 0$.

Thus, according to Fig. 7 and Eq. (1), the excitation of an arbitrarily directed lightning discharge is:

$\overline{\boldsymbol{J}}_{1}=\left|\overline{\boldsymbol{J}}_{1}\right| \sin \beta \overline{\boldsymbol{i}}+\left|\overline{\boldsymbol{J}}_{1}\right| \cos \beta \cos \alpha \overline{\boldsymbol{j}}+\left|\overline{\boldsymbol{J}}_{1}\right| \cos \beta \sin \alpha \overline{\boldsymbol{k}}$. (3)

We present the particular result of the general solution, applied here for tilted ground stroke cases, where the excitation is perpendicular to the propagation direction $(\beta=0)$.

The initial equations to be solved are the Maxwell's equations:

$$
\begin{aligned}
& \bar{\nabla} \times \overline{\boldsymbol{H}}=\overline{\boldsymbol{J}}_{1}+\varepsilon_{0} \frac{\partial \overline{\boldsymbol{E}}}{\partial t} \\
& \bar{\nabla} \times \overline{\boldsymbol{E}}=-\mu_{0} \frac{\partial \overline{\boldsymbol{H}}}{\partial t} \\
& \bar{\nabla} \overline{\boldsymbol{H}}=0 \\
& \bar{\nabla} \overline{\boldsymbol{E}}=\frac{\rho}{\varepsilon_{0}}
\end{aligned}
$$

where $\overline{\boldsymbol{H}}$ and $\overline{\boldsymbol{E}}$ are the magnetic and electric field strength vectors, $\overline{\boldsymbol{J}}_{1}$ is the exitation, $\varepsilon_{0}$ and $\mu_{0}$ are the vacuum permittivity and permeability.

The usual definition equations of the retarded potential is given:

$$
\begin{aligned}
& \bar{\nabla} \times \overline{\boldsymbol{A}}=\overline{\boldsymbol{H}} \\
& \overline{\boldsymbol{E}}+\mu_{0} \frac{\partial \overline{\boldsymbol{A}}}{\partial t}=-\bar{\nabla} \psi,
\end{aligned}
$$

where $\overline{\boldsymbol{A}}$ and $\psi$ are the common vector- and scalar-potentials, while the valid Lorentz condition is:

$$
\left(\bar{\nabla} \bar{A}+\varepsilon_{0} \frac{\partial \psi}{\partial t}\right)=0 .
$$

The equation of the vector-potential to be solved is:

$$
\nabla^{2} \overline{\boldsymbol{A}}-\varepsilon_{0} \mu_{0} \frac{\partial^{2} \overline{\boldsymbol{A}}}{\partial t^{2}}=-\overline{\boldsymbol{J}}_{1} .
$$

As the excitation is a general shaped signal with an exact starting point according to time and space, the Laplacetransformation can be applied as

$$
\begin{aligned}
t & \stackrel{L}{\leftrightarrow} s \\
x & \stackrel{L}{\leftrightarrow} p \\
y & \stackrel{\leftrightarrow}{\leftrightarrow} u \\
z & \stackrel{L}{\leftrightarrow} l \\
f(t, x, y, z) & \stackrel{L}{\leftrightarrow} F(s, p, u, l) .
\end{aligned}
$$

Due to the presence of derivative terms, initial values in all coordinates will appear. Usually these initial values contain information regarding the energetic state of the medium. However, in this case the medium is considered to be free of energy before the excitation. Therefore, in the following,

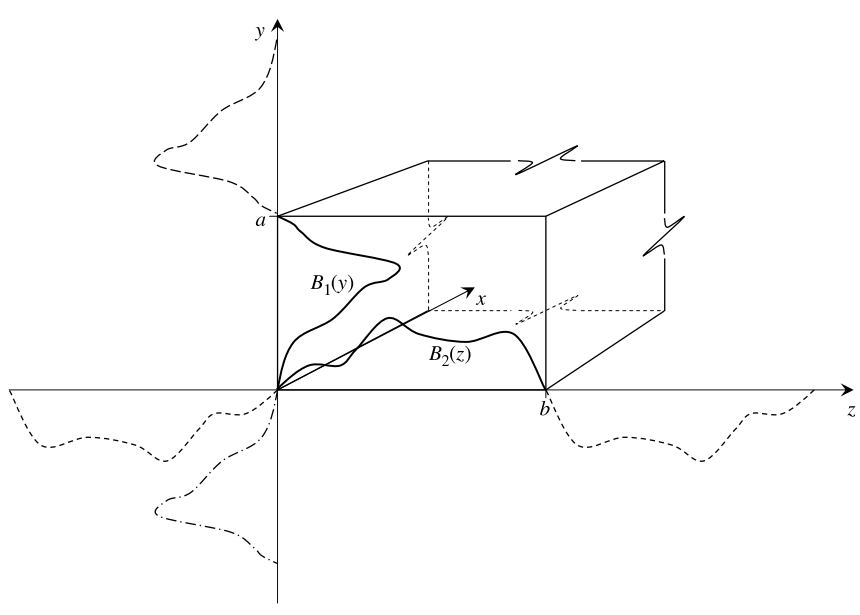

Fig. 8. Periodic extension of the source envelope functions.

all initial values have to be taken as zero. The transformed equations of the field components obtained from Eq. (7), to be solved are:

$H_{x}(s, p, u, l)=u A_{z}(s, p, u, l)-l A_{y}(s, p, u, l)$

$H_{y}(s, p, u, l)=-p A_{z}(s, p, u, l)$

$H_{z}(s, p, u, l)=p A_{y}(s, p, u, l)$,

further

$$
\begin{aligned}
& E_{x}(s, p, u, l)=\frac{1}{\varepsilon_{0}} \frac{1}{s}\left[p u A_{y}(s, p, u, l)+p l A_{z}(s, p, u, l)\right] \\
& \begin{aligned}
E_{y}(s, p, u, l) & =\frac{1}{\varepsilon_{0}} \frac{1}{s}\left[u^{2} A_{y}(s, p, u, l)+u l A_{z}(s, p, u, l)\right] \\
\quad-\mu_{0} s A_{y} & (s, p, u, l) \\
E_{z}(s, p, u, l) & =\frac{1}{\varepsilon_{0}} \frac{1}{s}\left[u l A_{y}(s, p, u, l)+l^{2} A_{z}(s, p, u, l)\right] \\
\quad-\mu_{0} s A_{z} & (s, p, u, l) .
\end{aligned}
\end{aligned}
$$

The Laplace transformed form of the exciting current density is

$$
\begin{aligned}
J_{1}(s, p, u, l)= & \iiint_{0}^{\infty} \int \delta(t) \delta(x) B_{1}(y) B_{2}(z) \\
& \cdot e^{-s t} \cdot e^{-p x} \cdot e^{-u y} \cdot e^{-l z} d t d x d y d z \\
= & B_{1}(u) B_{2}(l) .
\end{aligned}
$$

The proper choice of the $B_{1}$ and $B_{2}$ functions is important. These envelope functions contain the boundary conditions which resulted from the geometrical structure of the model. This can be seen in Fig. 8 .

The envelope functions $B_{1}$ and $B_{2}$ (see also in Eq. (2)) can be extended periodically, and it is possible to describe them by Fourier-series:

$$
\begin{aligned}
& B_{1}(y)=\sum_{m=0}^{\infty} C_{m} \cdot \mathrm{e}^{\mathrm{j} m \frac{\pi}{a} y} \\
& C_{m}=\frac{1}{2 a} \int_{-a}^{a} B_{1}(y) \cdot \mathrm{e}^{-\mathrm{j} m \frac{\pi}{a} y} \mathrm{~d} y
\end{aligned}
$$


and

$$
\begin{aligned}
& B_{2}(z)=\sum_{n=0}^{\infty} C_{n} \cdot \mathrm{e}^{\mathrm{j} n \frac{\pi}{b} z} \\
& C_{n}=\frac{1}{2 b} \int_{-b}^{b} B_{2}(z) \cdot \mathrm{e}^{-\mathrm{j} n \frac{\pi}{b} z} \mathrm{dz},
\end{aligned}
$$

where $C_{m}$ and $C_{n}$ are Fourier-coefficients, $a$ and $b$ are geometrical parameters of the waveguide, $m$ and $n$ are integers

$$
\begin{aligned}
& m=0, \pm 1, \pm 2, \ldots \\
& n=0, \pm 1, \pm 2, \ldots
\end{aligned}
$$

The Laplace-transformed forms of Eqs. (12) and (13) are

$$
\begin{aligned}
& B_{1}(u)=\sum_{m=-\infty}^{\infty} \frac{C_{m}}{u-\mathrm{j} m \frac{\pi}{a}} \\
& B_{2}(l)=\sum_{n=-\infty}^{\infty} \frac{C_{n}}{l-\mathrm{j} n \frac{\pi}{b} .}
\end{aligned}
$$

The non-zero poles according to $p$ can be determined from

$p^{2}+u^{2}+l^{2}-\varepsilon_{0} \mu_{0} s^{2}=0$

Investigating the infinite number of poles according to $u$ and $l$, by substituting Eq. (16) in Eqs. (9) and (10), it becomes obvious that only the poles originating from the excitation will yield terms different from zero in the amplitudes.

The well known steps of the inverse Laplacetransformation on the common way with the substitution:

$s=j \omega$

yields the needed field components of the guided wave. The obtained spectral forms of the space-dependent field components are:

$$
\begin{aligned}
H_{x}(\omega, x, y, z)= & \sum_{m} \sum_{n} \frac{P_{-} C_{m} C_{n}}{2 k_{x}(\omega)} \cdot e^{j\left[k_{x}(\omega) \cdot x \cdot M \cdot N\right]} \\
& -\sum_{m} \sum_{n} \frac{P_{-} C_{m} C_{n}}{2 k_{x}(\omega)} \cdot e^{j\left[-k_{x}(\omega) \cdot x \cdot M \cdot N\right]} \\
H_{y}(\omega, x, y, z)= & \sum_{m} \sum_{n} C_{m} C_{n} \sin \alpha \cdot e^{j\left[k_{x}(\omega) \cdot x \cdot M \cdot N\right]} \\
& +\sum_{m} \sum_{n} C_{m} C_{n} \sin \alpha \cdot e^{j\left[-k_{x}(\omega) \cdot x \cdot M \cdot N\right]} \\
H_{z}(\omega, x, y, z)= & \sum_{m} \sum_{n} C_{m} C_{n} \cos \alpha \cdot e^{j\left[k_{x}(\omega) \cdot x \cdot M \cdot N\right]} \\
& +\sum_{m} \sum_{n} C_{m} C_{n} \cos \alpha \cdot e^{j\left[-k_{x}(\omega) \cdot x \cdot M \cdot N\right]}
\end{aligned}
$$

$$
\begin{aligned}
E_{x}(\omega, x, y, z)= & \sum_{m} \sum_{n} \frac{-P_{+} C_{m} C_{n}}{2 \omega \varepsilon_{0}} \cdot e^{j\left[k_{x}(\omega) \cdot x \cdot M \cdot N\right]} \\
& +\sum_{m} \sum_{n} \frac{P_{+} C_{m} C_{n}}{(-2) \omega \varepsilon_{0}} \\
& \cdot e^{j\left[-k_{x}(\omega) \cdot x \cdot M \cdot N\right]} \\
E_{y}(\omega, x, y, z)= & \sum_{m} \sum_{n} \frac{\left[(-j m \pi / a)\left(j P_{+}\right)-\varepsilon_{0} \mu_{0} \omega^{2} \cos \alpha\right] C_{m} C_{n}}{2 j \varepsilon_{0} \omega j k_{x}(\omega)} \\
& \cdot e^{j\left[k_{x}(\omega) \cdot x \cdot M \cdot N\right]}+ \\
& +\sum_{m} \sum_{n} \frac{\left[(-j m \pi / a)\left(j P_{+}\right)-\varepsilon_{0} \mu_{0} \omega^{2} \cos \alpha\right] C_{m} C_{n}}{(-2) j \varepsilon_{0} \omega j k_{x}(\omega)} \\
& \cdot e^{j\left[-k_{x}(\omega) \cdot x \cdot M \cdot N\right]} \\
E_{z}(\omega, x, y, z)= & \sum_{m} \sum_{n} \frac{\left[(-j n \pi / b)\left(\mathrm{j} P_{+}\right)-\varepsilon_{0} \mu_{0} \omega^{2} \sin \alpha\right] C_{m} C_{n}}{2 \mathrm{j} \varepsilon_{0} \omega j k_{x}(\omega)} \\
& \cdot e^{j\left[k_{x}(\omega) \cdot x \cdot M \cdot N\right]}+ \\
& +\sum_{m} \sum_{n} \frac{\left[(-n \pi / b) P_{+}-\varepsilon_{0} \mu_{0} \omega^{2} \sin \alpha\right] C_{m} C_{n}}{(-2) j \varepsilon_{0} \omega j k_{x}(\omega)} \\
& \cdot e^{j\left[-k_{x}(\omega) \cdot x \cdot M \cdot N\right]},
\end{aligned}
$$

where

$P_{+}=m \frac{\pi}{a} \cos \alpha+n \frac{\pi}{b} \sin \alpha, \quad P_{-}=-m \frac{\pi}{a} \cos \alpha+n \frac{\pi}{b} \sin \alpha$,

$M=m \frac{\pi}{a} y, \quad N=n \frac{\pi}{b} z$,

$k_{x}(\omega)=\sqrt{\varepsilon_{0} \mu_{0} \omega^{2}-\left(m \frac{\pi}{a}\right)^{2}-\left(n \frac{\pi}{b}\right)^{2}}$.

It can be seen that one term in the field components propagates forward, the other propagates backward, considering the location of the excitation as a starting point $(x=0)$ within the assumed infinite long waveguide.

The limiting wavelength and thus, the limiting frequency can be obtained from Eqs. (18) and (19), yielding the same expressions as in the monochromatic approaches

$$
\begin{aligned}
& \varepsilon_{0} \mu_{0} \omega^{2}-\left(m \frac{\pi}{a}\right)^{2}-\left(n \frac{\pi}{b}\right)^{2}=0 \\
& \lambda_{m, n}=\frac{2 a b}{\sqrt{(m b)^{2}+(n a)^{2}}} .
\end{aligned}
$$

By the application of the formal inverse Fouriertransformation, the complete time- and space-dependent exact form of the propagating electric and magnetic 
field-components can be obtained as

$$
\begin{aligned}
H_{x}(t, x, y, z)= & \frac{1}{4 \pi} \int_{-\infty}^{\infty}\left\{\sum_{m} \sum_{n} \frac{P_{-} C_{m} C_{n}}{k_{x}(\omega)} \cdot e^{j T_{+}} \cdot e^{j M} \cdot e^{j N}\right. \\
& \left.-\sum_{m} \sum_{n} \frac{P_{-} C_{m} C_{n}}{k_{x}(\omega)} \cdot e^{j T_{-}} \cdot e^{j M} \cdot e^{j N}\right\} d \omega \\
H_{y}(t, x, y, z)= & \frac{1}{4 \pi} \int_{-\infty}^{\infty}\left\{\sum_{m} \sum_{n} C_{m} C_{n} \sin \alpha \cdot e^{j T_{+}} \cdot e^{j M} \cdot e^{j N}\right. \\
& \left.+\sum_{m} \sum_{n} C_{m} C_{n} \sin \alpha \cdot e^{j T_{-}} \cdot e^{j M} \cdot e^{j N}\right\} d \omega \\
H_{z}(t, x, y, z)= & \frac{-1}{4 \pi} \int_{-\infty}^{\infty}\left\{\sum_{m} \sum_{n} C_{m} C_{n} \cos \alpha \cdot e^{j T_{+}} \cdot e^{j M} \cdot e^{j N}\right. \\
& \left.+\sum_{m} \sum_{n} C_{m} C_{n} \cos \alpha \cdot e^{j T_{-}} \cdot e^{j M} \cdot e^{j N}\right\} d \omega \\
E_{x}(t, x, y, z)= & \frac{-1}{4 \pi} \int_{-\infty}^{\infty}\left\{\sum_{m} \sum_{n} \frac{P_{+} C_{m} C_{n}}{\omega \varepsilon_{0}} \cdot e^{j T_{+}} \cdot e^{j M} \cdot e^{j N}\right. \\
& \left.+\sum_{m} \sum_{n} \frac{P_{+} C_{m} C_{n}}{\omega \varepsilon_{0}} \cdot e^{j T_{-}} \cdot e^{j M} \cdot e^{j N}\right\} d \omega \\
= & \frac{1}{4 \pi} \int_{-\infty}^{\infty}\left\{\sum_{m} \sum_{n} \frac{\left[(-m \pi / a) P_{+}-\varepsilon_{0} \mu_{0} \omega^{2} \cos \alpha\right] C_{m} C_{n}}{\varepsilon_{0} \omega k_{x}(\omega)}\right. \\
& \cdot e^{j T_{+}} \cdot e^{j M} \cdot e^{j N}- \\
& -\sum_{m} \sum_{n} \frac{\left[(-m \pi / a) P_{+}-\varepsilon_{0} \mu_{0} \omega^{2} \cos \alpha\right] C_{m} C_{n}}{\varepsilon_{0} \omega k_{x}(\omega)} \cdot e^{j T_{-}} \\
& \left.\cdot e^{j M} \cdot e^{j N}\right\} d \omega \\
E_{y}(t, x, y, z) & \left.\cdot e^{j M} \cdot e^{j N}\right\} d \omega, \\
& \cdot \int_{-\infty}^{j T_{+}}\left\{e_{m}^{j M} \cdot \sum_{n} \frac{\left[(-n \pi / b) P_{+}-\varepsilon_{0} \mu_{0} \omega^{2} \sin \alpha\right] C_{m} C_{n}}{\varepsilon_{0} \omega k_{x}(\omega)}\right. \\
& -\sum_{m} \sum_{n} \frac{\left[(-n \pi / b) P_{+}-\varepsilon_{0} \mu_{0} \omega^{2} \sin \alpha\right] C_{m} C_{n}}{\varepsilon_{0} \omega k_{x}(\omega)} \cdot e^{j T_{-}} \\
E_{z}(t, x, y, z) & \left.\frac{1}{4 \pi}\right)
\end{aligned}
$$

where

$T_{+}=\omega t+k_{x}(\omega) \cdot x \quad T_{-}=\omega t-k_{x}(\omega) \cdot x$.

Tweeks, as guided propagating impulses, can excite the lower ionosphere, generating subsequent upward wave propagation in the anisotropic, inhomogeneous plasma. The detailed description of the analytical real UWB solution of signal propagation in an arbitrarily inhomogeneous anisotropic plasma can be found in Ferencz (2005), based on the results of the solution of monochromatic propagation (Ferencz, $2004 b$ ). We concentrate in the following on the results of numerical simulations of subionospheric tweek waveforms and on modeled SpW signals excited by tweeks, according to the schematic in Fig. 6, without repeating the published results on a full-wave solution of impulse propagation in the inhomogeneous plasma.

\section{Tweek and SpW: numerical results of a full-wave so- lution, comparison of modeling and observation}

The application of the closed form, full-wave solution of guided wave propagation, summarized in Ferencz (2004a) and discussed in Sect. 4 yields the accurate time function of any e.m. field component, representing the signal measured at any point in the EIWG. UWB analysis and mod-
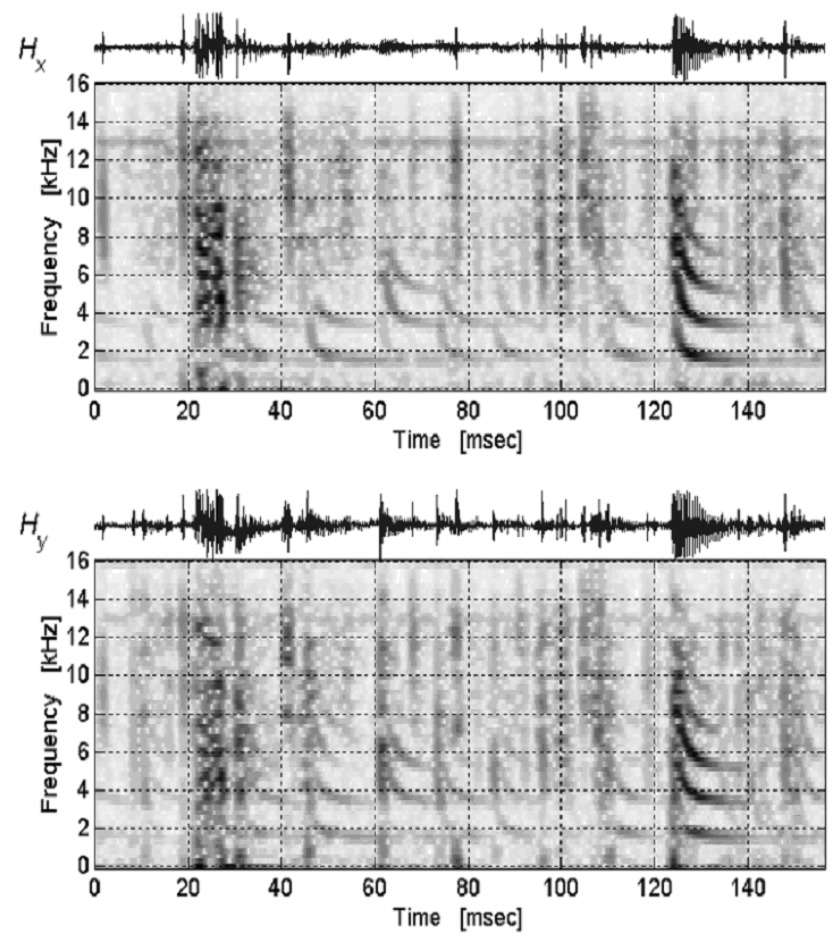

Fig. 9. Series of tweeks in a cut of a two-channel, crossedloop broadband VLF recording at Marion Island (22 April 2001, 02:30:07 UT). Tweeks in the above panel (NS oriented loop) are appearing without the TEM mode, in agreement with an almost meridional spheric propagation to the receiver according to our modelling (with kind permission of A. R. W. Hughes, Durban, South Africa).

elling of tweeks, recorded using crossed loop magnetic antennae shows that the signal pattern of the orthogonal components carries information not only about the "ionospheric height" and the length of the propagation path, as previous (monochromatic) approaches do, but about the propagation direction as well. According to Eq. (21) the signal amplitude of the basic (lowest or TEM) mode, best seen on spectograms at frequencies below the 1 st harmonic, depends on the incident angle at the receiving point. In the particular case when the propagation direction (axis $x$ in our model, see Figs. 7 and 8) falls close to the plane of one loop, the two perpendicular components have a remarkably different shape: the basic mode may almost miss one component, while exhibiting maximum intensity on the other. This circumstance, illustrated in observation data in Figs. 9, 11 and in model calculations seen in Fig. 10, supplies an efficient tool to determine the incident angle accurately by choosing the directions of the $x$ and $y$ model axes properly with respect to the observed data. Furthermore, the option to vary the directions of the source (lightning discharching channel) enables more realistic investigation of tweeks than previous methods do.

The subsequent propagation of an impulse in the magnetoionic medium, excited by a tweek at the lower ionosphere was modelled. For this step the analytical UWB solution 
of wave propagation in case of arbitrarily inhomogeneous plasmas (Ferencz, 2005), or the full wave solution of an oblique impulse propagation (Ferencz et al., 2001), can be applied. Note that the waveforms of short-path fractionalhop whistlers, acquired at LEO altitudes, are most accurately described assuming an oblique, nonducted propagation in the ionospheric plasma. Our model calculations illustrated in this paper were based on medium characteristic, derived from the standard models, IRI (Bilitza, 2001) and IGRF (Bilitza and Papitashvili, 2006).

\section{Discussion and conclusion}

The convincing agreement between observed and modelled SpWs confirm the validity of the described generation mechanism of these type of signals: SpWs are identified as specific short-path fractional-hop whistlers, excited by tweeks. Tweeks may also play an important role in whistler generation in the widely accepted function of the same way as spheric impulses, if a fraction of the subionospherically propagating energy enters the plasmasphere and traverses the anisotropic medium. In the latter case, whistlers (SpWs) carry information at the same time about the signal generation, about the propagation conditions in the EIWG and about the anisotropic plasma in the ionosphere and magnetosphere along the propagation path.

After the recognition and a future regular (automatic) detection of the SpW signals, the accurate UWB modelling and interpretation provides a sensible tool to monitor the medium characteristic in the lower ionized environment of the Earth. The minimum "height" of the ionosphere along the path of the tweek and the local variations in plasma parameters during propagation across the magnetoionic medium can be traced at the same time. This approach may add a new aspect to global e.m. monitoring based on LEO satellites, with relevance for example, to space-weather and/or seismic processes, affecting existing or possible plasma conditions in the upper atmosphere. (There are indications that ionospheric perturbations, fluctuations in plasma conditions are associated with seismicity.)

Analysis of SpWs exhibiting an incomplete sequence of mode pattern, together with the interpretation of synchronized tweeks recorded on the ground, helps in better understanding the process how the energy of the guided signal, propagating along the Earth's surface, enters the plasma. The signal structure itself, local inhomogenities or other yet not revealed processes in the mesosphere may play important role in the out-coupling mechanism.

The signal pattern characteristic to the SpWs neither has been observed visually nor demonstrated with detailed fine structure analysis (Hamar and Tarcsai, 1982; Hamar et al., 1990) on any one-hop whistler, which was crossing the magnetosphere along the geomagnetic field lines and thus long propagating in the lossy anisotropic plasma. One proba- ble explanation is that signals excited by the basic (TEM) guided mode can only propagate through the magnetosphere in quasi-longitudinal propagation and/or can enter back to the lower atmosphere at the conjugate area of the source region. This interpretation fits well our experience on whistler analysis, however, we should note that the known UWB solutions of the Maxwell's equations cannot confirm this. Therefore, it would be effective and important, possibly to derive new UWB solutions of Maxwell's equations by applying, for example, more detailed boundary conditions, of a new description of wave-medium interactions. After the low occurrence rate of $\mathrm{SpWs}$ a future fine structure analysis of mass volume one-hop whistlers may yield new recognition regarding this particular point.

Triggered emissions, observed on the ground and in space with whistlers propagated in the magnetosphere, may exhibit similar branching patterns on spectograms. The clear asymptotic behaviour, the frequency of the observed asymptotes and the curvilinear character of the SpWs, observed on short-path fractional-hop whistlers undoubtedly separates them from triggered emissions. In contrary, we shall note that the almost monochromatic asymptoted signal part may play a significant role in a resonant wave-plasma interaction, possibly causing emission in the plasmasphere. This aspect has to be modelled and analyzed in later investigations.

Results of our analysis and full-wave modelling of SpW signals prove that lightning generated strong impulses can leak out the EIWG, and thus excite SpWs even after several thousand of $\mathrm{km}$ of subionospheric propagation. This fits well with the recent conclusion of the comparative, statistical study of European lightning data and conventional fractionalhop whistlers, recorded on board (Chum et al., 2006), estimating the source - subsatellite point distances much above one thousand $\mathrm{km}$.

Based on comparisons of lightning data and one-hop whistlers, detected automatically on continuous ground recordings it was concluded (Lichtenberger et al., 2005; Collier et al., 2006) that the primary source of whistlers are cloud-to-cloud (CC) lightnings and only a minor part of the whistler events are thought to excited by cloud-to-ground (CG) discharges, also discussed in Sect. 4, in a theoretical point of view. The observed preliminary ratio of the $\mathrm{SpW}$ and conventional fractional-hop whistler appearance, mentioned in Sect. 3, agrees with the known dominance of the $\mathrm{CC}$ discharges. However, we should stress that the number of lightnings, selected in a reasonably large region around the satellite observation, are typically much higher than the occurrences of whistlers. This means that lightning is only one necessary element in whistler generation. This is even more important in the case of SpWs, where the formation of tweeks depends not only on excitation character but on subionospheric propagation properties (e.g. ground surface conductivity, ionisation profile in the mesosphere).

After the stage of this first report of a new observation a systematic future analysis of SpWs measured by different 

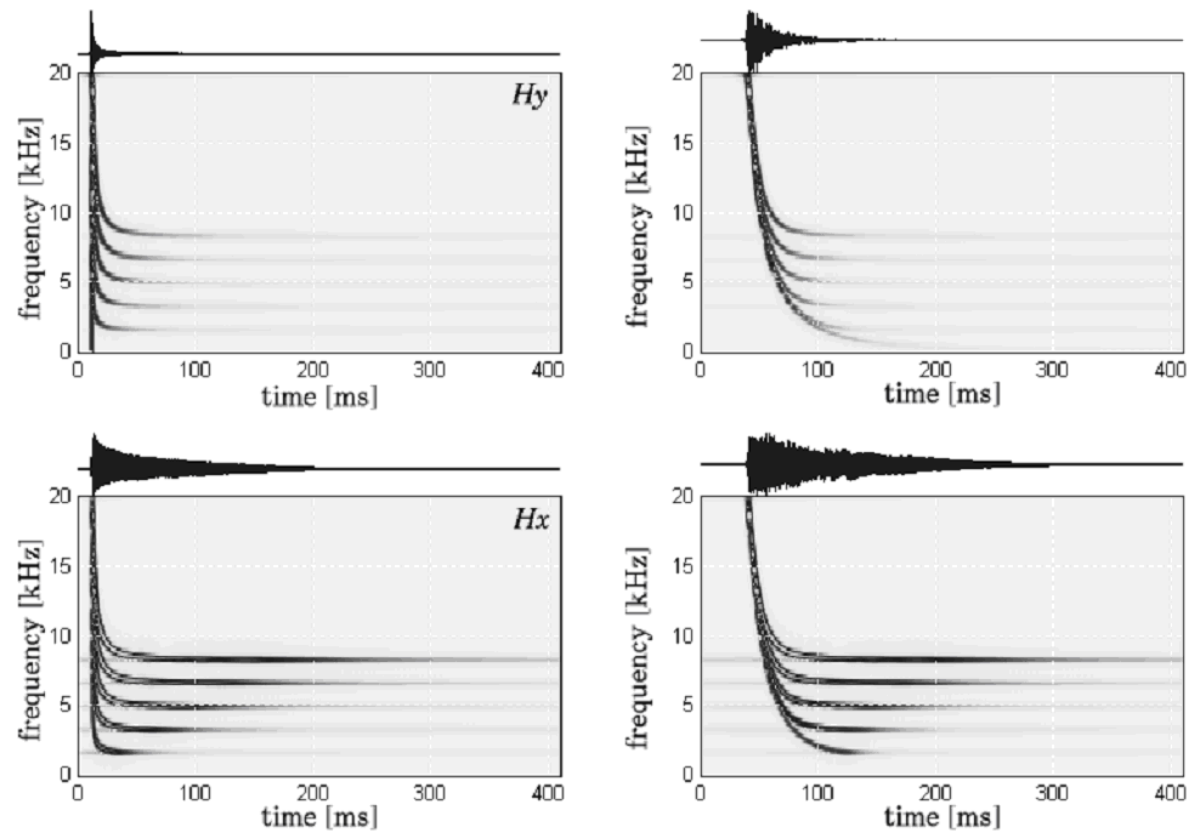

Fig. 10. Results of real full-wave modelling of long spheric propagation in the EIWG, appearing as a tweek, with and without the basic TEM mode, at a ground receiving station's loop (left column) and of a subsequent impulse propagation across the inhomogeneous ionosphere, forming $\mathrm{SpW}$ signals, observable on board the LEO satellites. (This model calculation was assuming Dirac excitation, 3500-km long propagation along the $x$ axis in a vacuum-filled EIWG and a vertical propagation across the mid-latitude ionosphere to a LEO satellite at $700 \mathrm{~km}$ altitude.)
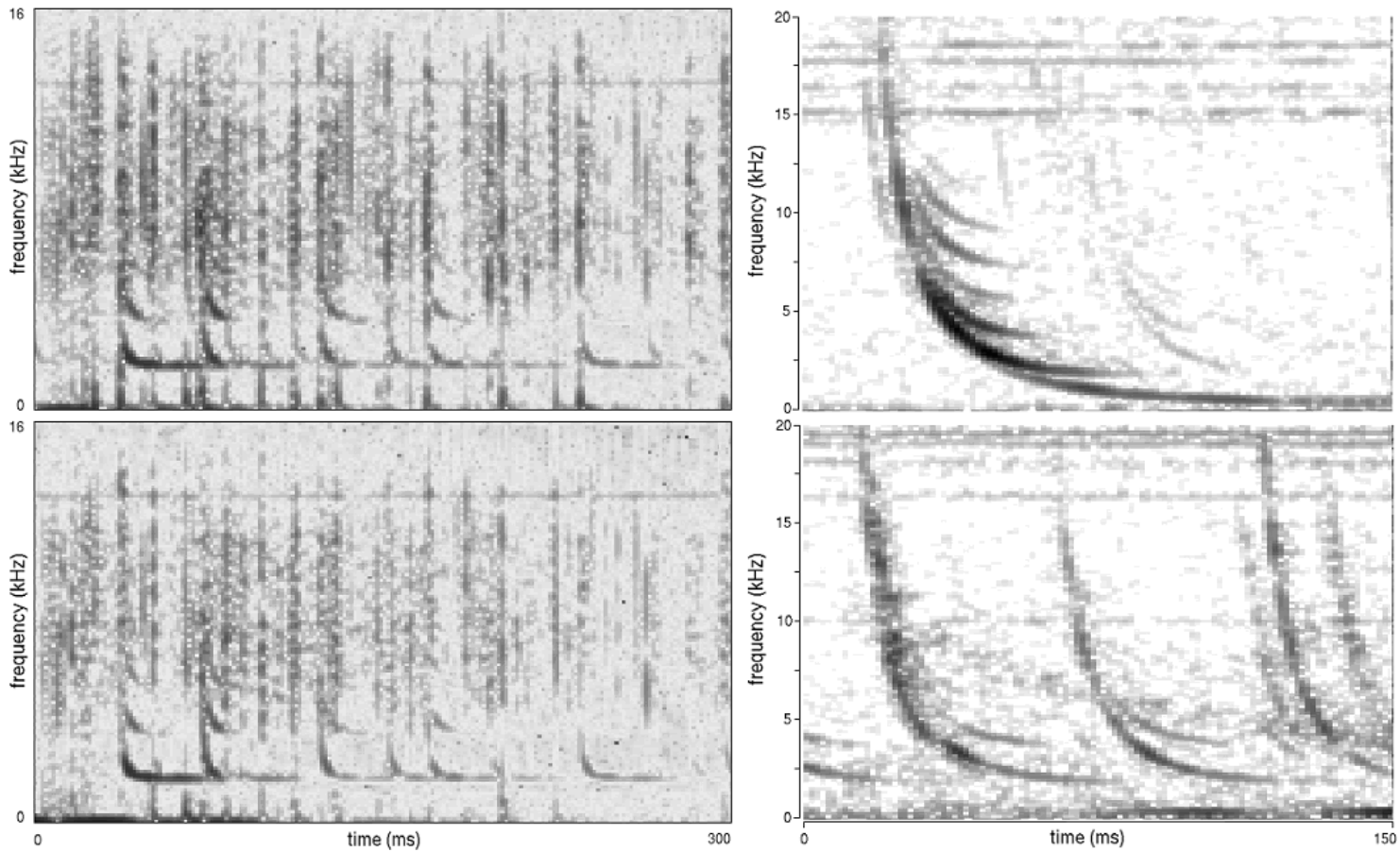

Fig. 11. Tweeks (left column) in a ground-based, crossed loop VLF recording (Marion Island, 28 April 2001, 03:15:25 UT, with permisson of A. R. W. Hughes, Durban, South Africa) and examples of SpWs (DEMETER VLF ICE, right column) exhibiting similar mode patterns. 
satellites at different, higher orbit altitudes is needed. That investigation could clarify several aspects and still open questions of SpW signals.

Acknowledgements. Authors are grateful to the Guest Investigator program issued by CNES for the DEMETER mission for supplying the raw data. The projects TP 276 of the Hungarian Space Office and OTKA T037611 and F037603 of the Hungarian National Scientific Fund are acknowledged.

Topical Editor M. Pinnock thanks two referees for their help in evaluating this paper.

\section{References}

Berthelier, J. J., Godefroy, M., Leblanc, F., Malingre, M., Menvielle, M., Lagoutte, D., Brochot, J. Y., Colin, F., Elie, F., Legendre, C., Zamora, P., Benoist, D., Chapuis, Y., and Artru, J.: ICE, the electric field experiment on DEMETER, Planet. Space Sci, 54(5), 456-471, 2006.

Bilitza, D.: International Reference Ionosphere 2000, Radio Sci., 36(2), 261-275, 2001.

Bilitza, D. and Papitashvili, N.: SSCWeb and ModelWeb updates: IGRF 10 and more, AGU SPA Section Newsletter, XIII, 12, 2006.

Budden, K. G.: The wave-guide mode theory of wave propagation, Logos Press, London, 1961.

Burke, C. P. and Jones, D. L.: An experimental investigation of ELF attenuation rates in the Earth-ionosphere waveguide, J. Atmos. Terr. Phys., 54, 243-250, 1992.

Chum, J., Jiřiček, F., Santolik, O., Parrot, M., Diendorfer, G., and Fiser, J.: Assigning the causative lightning to the whistlers observed on satellites, Ann. Geophys, 24, 2921-2929, 2006.

Collier, A. B., Hughes, A. R. W., Lichtenberger, J., and Steinbach, P.: Seasonal and diurnal variation of lightning activity over southern Africa and correlation with European whistler observations, Ann. Geophys., 24, 529-542, 2006,

http://www.ann-geophys.net/24/529/2006/.

Cummer, S. A., Inan, U. S., and Bell, T. F.: Ionospheric D region remote sensing using VLF radio atmospherics, Radio Sci., 33(6), 1781-1792, 1998.

Ferencz, Cs., Bognár, P., Tarcsai, Gy., Hamar, D., and Smith, A. J.: Whistler-mode propagation: results of model calculations for an inhomogeneous plasma, J. Atmos. Terr. Phys., 58, 625-640, 1996.

Ferencz, Cs., Ferencz, O. E., Hamar, D., and Lichtenberger, J.: Whistler Phenomena, Short Impulse Propagation, Kluwer Academic Publishers, Dordrecht, 2001.
Ferencz, O. E.: Short impulse propagation in wave-guides; Híradástechnika "Communications", LIX, No. 6, 2-6, 2004a.

Ferencz, Cs.: Real solution of monochromatic wave propagation in inhomogeneous media, Pramana J. Phys., 62, 943-955, 2004 b.

Ferencz, Cs. and Ferencz, O. E.: Impulse propagation problems and models in space and communication systems (invited), The 8th World Multi-Conference on Systemics, Cybernetics and Informatics, 18-21 July 2004, Orlando, Florida, USA, XI, 172-179, 2004.

Ferencz, O. E.: Full-wave solution of short impulses in inhomogeneous plasma, Pramana J. Phys., 64, 249-268, 2005.

Hamar, D. and Tarcsai, Gy.: High resolution frequency-time analysis using digital matched filtering. Part I: Theory and simulation studies, Ann. Geophys., 38, 119-128, 1982, http://www.ann-geophys.net/38/119/1982/.

Hamar, D., Tarcsai, Gy., Lichtenberger, J., Smith, A. J., and Yearby, K. H.: Fine structure of whistlers recorded digitally at Halley, Antarctica, J. Atmos. Terr. Phys, 52, 801-810, 1990.

Hayakawa, M., Ohta, K., and Baba, K.: Wave characteristics of tweek atmospherics deduced from the direction-finding measurement and theoretical interpretation, J. Geophys. Res., 99(D5), 10733-10 743, 1994.

Helliwell, R. A.: Whistlers and related ionospheric phenomena, 349 pp., Stanford University Press, Stanford, California, 1965.

Holden, D. N., Munston, C. P., and Davenport, J. C.: Satellite observations of transionospheric pulse pairs, Geophys. Res. Lett., 22, 889-892, 1995.

Lichtenberger, J., Bodnár, L., Ferencz, Cs., Ferencz, O. E., Hamar, D., and Steinbach, P.: Automatic whistler detector, First results, IAGA IASPEI, G2.07, 2001 Hanoi, p.124, 2001.

Lichtenberger, J., Hamar, D., Ferencz, Cs., Ferencz, O. E., Collier, A., and Hughes, A.: What are the sources of whistlers?, URSI XVIII. Gen Ass., H.2, New Delhi, India, 2005.

Parrot, M., Benoist, D., Berthelier, J. J., Bêcki, J., Chapuis, Y., Colin, F., Elie, F., Fergeau, P., Lagoutte, D., Lefeuvre, F., Legendre, C., Lévêque, M., Pinçon, J. L., Poirier, B., Seran, H. C., and Zamora, P.: The magnetic field experiment IMSC and its data processing onboard DEMETER: scientific objectives, description and first results, Planet. Space Sci., 54(5), 441-455, 2006.

Shvets, A. V. and Hayakawa, M.: Polarisation effects for tweek propagation, J. Atmos. Sol-Ter. Phys., 60(4), 461-469, 1998.

Simonyi, K.: Foundation of Electrical Engineering, Pergamon Press, New York, 1963.

Taylor, W. L.: VLF attenuation for east-west and west-east daytime propagation using atmospherics, J. Geophys. Res., 65, 19331938, 1960.

Yedemsky, D. Ye., Ryabov, B. S., and Shchokotov, A. Yu.: Experimental investigation of the tweek field structure, Adv. Space Res., 12(6), 251-254, 1992. 